\title{
Improvement of bone defect healing in rats via mesenchymal stem cell supernatant
}

\author{
WANSHAN ZHOU ${ }^{1 *}$, QIAN LIU ${ }^{2 *}$ and $\mathrm{BO} \mathrm{XU}^{2}$ \\ Departments of ${ }^{1}$ Surgery and ${ }^{2}$ Orthopedics, Dezhou People's Hospital, Dezhou, Shandong 253014, P.R. China
}

Received July 20, 2017; Accepted November 2, 2017

DOI: $10.3892 /$ etm.2017.5534

\begin{abstract}
The effects of mesenchymal stem cells (MSCs) from three different sources in the treatment of bone defect with stem cells, and the differences of curative effects were studied. The umbilical cord, adipose and bone marrow mesen-chymal stem cells (BMSCs) of Sprague-Dawley (SD) rats were isolated and extracted, and the phenotype was identified for the 4th generation. The SD rat model of bone defect was established. The rats were randomly divided into: Normal saline group, umbilical cord mesenchymal stem cell (UMSC) group, adipose mesenchymal stem cell (AMSC) group and BMSC group. Rats were treated with tail intravenous injection, followed by radiological examination. The relative expression levels of factors bone morphogenetic protein-2 (BMP-2), osteocalcin (OCN), alkaline phosphatase (ALP), sclerostin (SOST), collagen carboxy-terminal telopeptide (CTX) and tartrated resistant acid phosphatase (TRACP) were measured via fluorescence quantitative PCR and western blotting. Among the three different kinds of stem cell supernatant, the detection using bicinchoninic acid (BCA) method showed that the content of P4-generation new cytokines was the highest. Wound healing in the three stem cell supernatant groups was significant at 3 weeks after operation, which was faster than that in DF12 control group; the expression levels of BMP-2, OCN and ALP in the bone samples treated with three kinds of MSC supernatants after 5 weeks were significantly increased compared with those in control group. The expression levels of SOST, CTX and TRACP were significantly decreased compared with those in control group. Three kinds of MSC supernatants can promote the bone regeneration through promoting the secretion of relatively more osteoblast factors, and inhibit the bone loss. The concentration of cytokines in UMSC supernatant
\end{abstract}

Correspondence to: $\mathrm{Dr} \mathrm{Bo} \mathrm{Xu}$, Department of Orthopedics, Dezhou People's Hospital, 1751 Xinhu Avenue, Decheng, Dezhou, Shandong 253014, P.R. China

E-mail: xubo0987@126.com

*Contributed equally

Key words: mesenchymal stem cells, stem cell supernatant, bone defect, repair, relative expression level was the highest under the same culture condition, and BMSC supernatant has a better effect in improving the bone defect repair of rats under the same concentration of cytokines.

\section{Introduction}

With the advent of precision medicine, every country has paid increasing attention to the development and application of individualized treatment, and cell therapy has also gradually become the main method of individualized treatment with the capital injection and improvement of R\&D level. The stem cell treatment means in cell therapy is currently the field where each country in the world compete to study and make significant progress $(1,2)$. In particular, considerable progress has been made in the treatment of ophthalmic diseases, neurological diseases and bone defect repair (3). The treatment of bone defect mostly adopts scaffold materials or direct intravenous injection of stem cells, but there is little research on the specific components and repair mechanism (4). The stem cell supernatant contains a number of beneficial factors secreted to adapt to the external environment in the cell growth, and these factors can protect and regulate the damage of tissues and organs (5). Bone defect is the most common symptom in orthopedic diseases, which is mainly treated with bone grafting at present $(6,7)$. In this experiment, the concentrations of cytokines in the three kinds of different mesenchymal stem cell (MSC) supernatants and the rates of bone defect repair were compared to investigate whether the MSC supernatant, as a factor of stem cell therapy, can promote the bone defect repair.

\section{Materials and methods}

Materials and reagents. The following were used: P1-generation umbilical cord mesenchymal stem cells (UMSCs), P1-generation adipose mesenchymal stem cells (AMSCs), and P1-generation bone marrow mesenchymal stem cells (BMSCs) (all from ATCC, Manassas, VA, USA); BCA detection kit (TransGen, Beijing, China); F12/Dulbecco's modified Eagle's medium (DMEM) (Hyclone, Logan, UT, USA); 36 Sprague-Dawley (SD) rats, bone morphogenetic protein-2 (BMP-2), osteocalcin (OCN), alkaline phosphatase (ALP), sclerostin (SOST), $\beta$-CrossLaps, tartrated resistant acid phosphatse (TRACP) primers (Sangon, Shanghai, China); TRIzol (Invitrogen Life Technologies, Carlsbad, CA, USA); reverse transcription kit (Takara Bio, Inc., Otsu, China); rabbit 
anti-rat BMP-2, OCN polyclonal antibody (dilution, 1:500; cat. nos. sc-9003 and sc-30044; Santa Cruz Biotechnology, Inc., Santa Cruz, CA, USA), rabbit anti-rat polyclonal antibody (dilution, 1:1,000; cat. no. 11187-1-AP; Proteintech, Wuhan, China), HRP goat anti-rabbit polyclonal antibody (dilution, 1:2,000; cat. no. sc-2004; Santa Cruz Biotechnology, Inc.). The study was approved by the Ethics Committee of Dezhou People's Hospital.

Methods. Collection of stem cell supernatant and determination of cytokine content (8): P1-generation stem cells were sub-cultured in $75 \mathrm{~T}$ culture flask at $8000 / \mathrm{T}$ for $48 \mathrm{~h}$ until the P4 generation. Then the F12/DMEM containing serum was replaced with the serum-free medium, and the cells continued to be cultured for 3 days, followed by the collection of supernatant. Supernatant $(1 \mathrm{ml})$ was taken and the concentration of cytokines was detected using the bicinchoninic acid (BCA) method. Formula: Cytokine concentration = total protein concentration - protein concentration in DF12 medium.

Establishment of rat model of bone defect and drug administration (9). Thirty-six rats weighing 300-400 g were randomly divided into 4 groups with 9 rats in each group. Rats were kept in cages with controlled temperature and light cycles ( $24^{\circ} \mathrm{C}$ and $12 / 12$ light cycles) with free access to water. Under the anesthesia with ketamine $(60 \mathrm{mg} / \mathrm{kg})$, rats were fixed with a fixator, and $8 \mathrm{~mm}$ long middle section of femoral shaft with periosteum was removed. The collagen complex with stem cell supernatant freeze-dried powder containing $3 \mathrm{mg}$ cytokine was implanted into each rat. Rats were divided into: i) Control group; ii) UMSC supernatant group; iii) AMSC supernatant group; and iv) BMSC supernatant group.

Evaluation of bone defect healing effect (10). The bone healing was observed via computed tomography (CT) at 1, 3 and 5 weeks. Evaluation criteria of bone formation: No bone formation, 0 point; $25 \%$ bone formation, 1 point; $25-50 \%$ bone formation, 2 points; $51-75 \%$ bone formation, 3 points; $76-99 \%$ bone formation, 4 points; and $100 \%$ new bone filling the bone defect, 5 points.

The relative expression levels of BMP-2, OCN, ALP, SOST, CTX and TRACP in bone tissues of the 4 groups were detected via quantitative polymerase chain reaction (qPCR) after 6 weeks. The bone tissues at the bone defect were ground into powder, and the mRNA was extracted from bone cells using TRIzol. After reverse transcription, the detection was performed via qPCR amplification. According to the mRNA sequence of NCBI, Primer 5.0 was used to design the real-time primer sequences: BMP-2 forward, GAAGAGAAAAGCGT CAAGCGA and reverse, TGATCAGCCAGGGGAAAGG; OCN forward, AGGGCAGCGAGGTAGTGA and reverse, CCTGAAAGCCGATGTGGT; ALP forward, CAGTTAGAT TTGGAGGTTCAACTTC and reverse, GAAGTTGAACCT CCAAATCTAACTG; SOST forward, ATTCAGTGCCAA GGTCA and reverse, TGGAGGTAGCCAAACAT; CTX forward, AAGGTGTTGTGCGATGAC and reverse, GTCGG TGGGTGACTCTG; TRACP forward, AGCCCTTTCTAC CGC and reverse, CACGCCATTCTCATCTT; GAPDH forward, GGAGCGAGATCCCTCCAAAAT and reverse, GGCTGTTGTCATACTTCTCATGG.

The protein expression levels of BMP-2, osteogencin, ALP, SOST, CTX and TRACP were detected via western

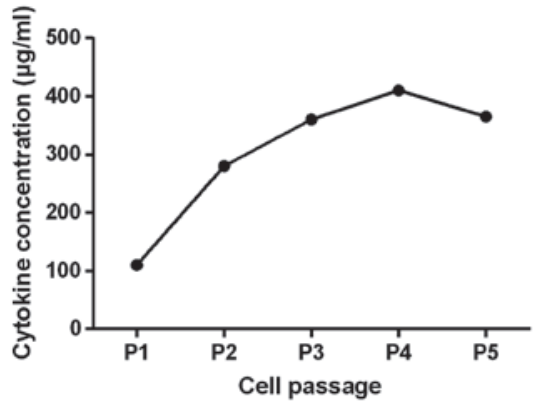

Figure 1. Cytokine concentrations in UMSC supernatant of P1-P5. UMSC, umbilical cord mesenchymal stem cell.

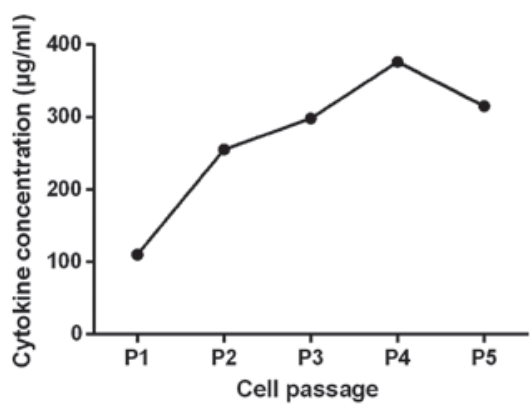

Figure 2. Cytokine concentrations in AMSC supernatant of P1-P5. AMSC adipose mesenchymal stem cell.

blotting. The lung tissues were ground in the liquid nitrogen and then transferred to RIPA protein lysis buffer for splitting on ice for $30 \mathrm{~min}$, during which the buffer was vibrated and mixed for 3 times. After centrifugation at $12,000 \mathrm{x}$ g at $4^{\circ} \mathrm{C}$ for $10 \mathrm{~min}$, the supernatant was placed in $1.5 \mathrm{ml}$ EP tube. Some supernatants were taken for detection of protein concentration using the BCA method, while some for ensuring that the protein content in each sample to be tested was $100 \mu \mathrm{g}$. Reduced loading buffer (5X) was added and boiled in boiling water for $10 \mathrm{~min}$. The above sample solution was slowly added into the sample well of the prepared gel with a micro-syringe. After SDS-PAGE under $80 \mathrm{~V}$, the target protein in the gel was transferred onto the $\mathrm{NC}$ membrane via the wet transfer under $40 \mathrm{~V}$ for $0.5 \mathrm{~h}$. After that, the protein was rinsed with eluent for $10 \mathrm{~min}$ (at least 3 times) and the antibody was given. Then the protein was sealed with skimmed milk powder at $4^{\circ} \mathrm{C}$ overnight. At room temperature, the primary antibody was incubated for $2 \mathrm{~h}$, and the secondary antibody was incubated for $1 \mathrm{~h}$; then the fluorescent substrate was added for tabletting and imaging in the dark.

Statistical analysis. Data were presented as mean \pm standard deviation, and SPSS 18.0 (SPSS, Inc., Chicago, IL, USA) software was used to analyze the one-way analysis of variance or t-test among groups. $\mathrm{P}<0.05$ was considered to indicate a statistically significant difference.

\section{Results}

Cytokine concentrations in the three different kinds of MSC supernatant. Among the three different kinds of stem cell supernatants, the detection using BCA method showed that 


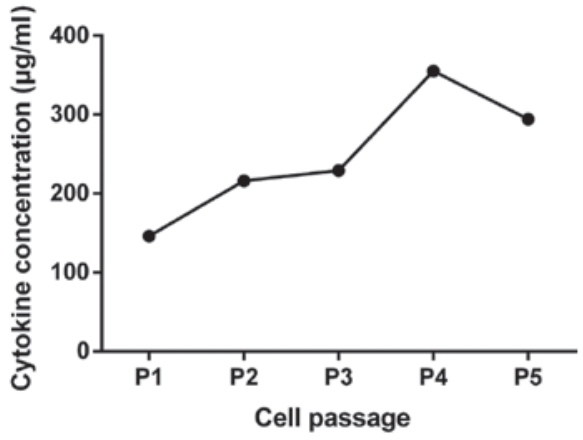

Figure 3. Cytokine concentrations in BMSC supernatant of P1-P5. BMSC, bone marrow mesenchymal stem cell.

Table I. Radiological score of bone formation in each group at different time-points after operation $(n=9)$.

\begin{tabular}{llll}
\hline Groups & Week 1 & Week 3 & Week 5 \\
\hline Control & $0.61 \pm 0.13$ & $0.95 \pm 0.11$ & $1.55 \pm 0.21$ \\
UMSC & $1.24 \pm 0.11^{\mathrm{a}}$ & $2.43 \pm 0.23^{\mathrm{a}}$ & $3.54 \pm 0.18^{\mathrm{a}}$ \\
AMSC & $1.32 \pm 0.43^{\mathrm{a}}$ & $2.32 \pm 0.31^{\mathrm{a}}$ & $3.32 \pm 0.19^{\mathrm{a}}$ \\
BMSC & $1.65 \pm 0.31^{\mathrm{b}}$ & $2.97 \pm 0.12^{\mathrm{b}}$ & $4.02 \pm 0.22^{\mathrm{b}}$ \\
\hline
\end{tabular}

${ }^{\mathrm{a} C}$ Compared with DF12 group, $\mathrm{p}<0.05$; ${ }^{\mathrm{b}}$ compared with UMSC group, $\mathrm{p}<0.05$. UMSC, umbilical cord mesenchymal stem cell; AMSC, adipose mesenchymal stem cell; BMSC, bone marrow mesenchymal stem cell.

the content of P4-generation new cytokines was the highest, then UMSC $(414 \mu \mathrm{g} / \mathrm{ml})>\operatorname{AMSC}(382 \mu \mathrm{g} / \mathrm{ml})>$ BMSC $(356 \mu \mathrm{g} / \mathrm{ml})$ (Figs. 1-3).

Effect of stem cell supernatant on bone healing. UMSC, AMSC and BMSC groups were detected via X-ray. The results revealed that porosis could be seen at 3 weeks after operation. At 5 weeks after operation, 3 out of 9 cases of bone defect were healed, and the healing effect in BMSC group was the best. Slight poroma could be seen in DF12 group, and the bone defect was not healed. At 5 weeks after operation, the porosis and wound healing were greatly improved in the three groups of stem cell supernatants, and large pieces of complete new bone appeared containing more lamellar bones and fibrous bones. There was no significant change in control group, and no poroma was found, only slight bone formation in the distal and proximal ends (Table I).

Detection of effect of MSC supernatant on the expression of osteoblast factors, BMP-2, OCN and ALP, in bone defect via $q P C R$ and western blotting. At 5 weeks after operation, the activities of BMP-2, OCN and ALP in bone cells were significantly increased after treatment with the three kinds of stem cell supernatants, and the expression levels under BMSC supernatant treatment were the highest. MSC supernatant, especially BMSC supernatant, could significantly promote the expressions of osteoblast factors, thus promoting the bone defect healing (Fig. 4).

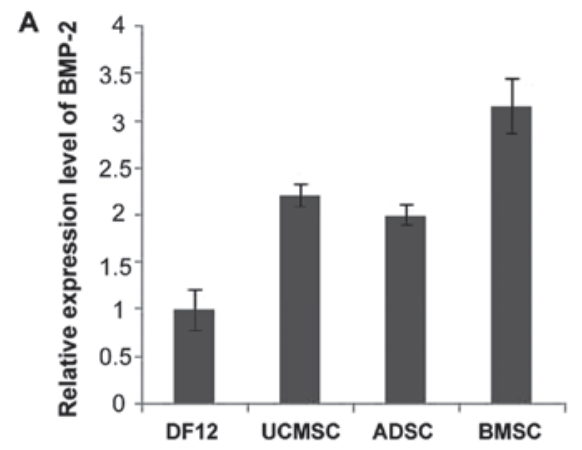

B
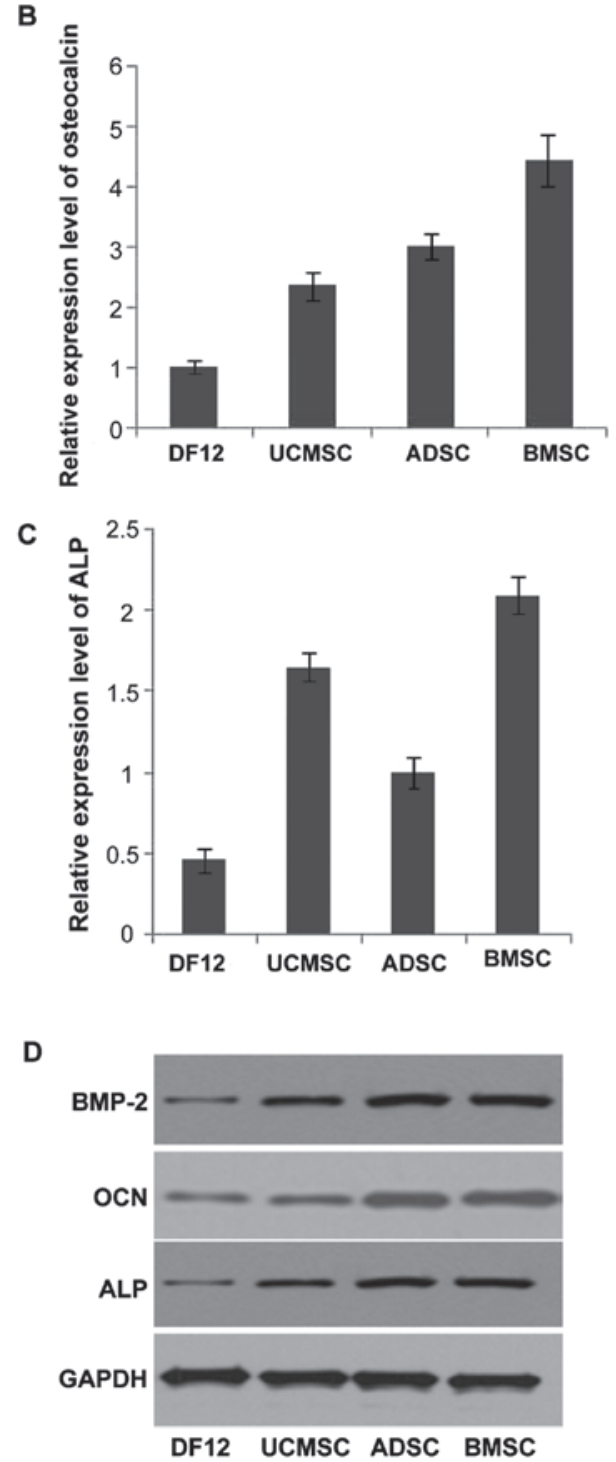

Figure 4. Effect of MSC supernatant on the expression of osteoblast factors in bone defects. (A-C) Detection of the relative expression levels of BMP-2, OCN and ALP via qPCR. (D) Detection results of western blotting. MCS, mesenchymal stem cell; BMP-2, bone morphogenetic protein-2; OCN, osteocalcin; ALP, alkaline phosphatase.

Detection of effect of MSC supernatant on the expression of factors related to the inhibition of bone formation and resorption, SOST, CTX and TRACP, in bone defect via qPCR and western blotting. At 5 weeks after operation, the activities of SOST, CTX and TRACP in bone cells were significantly decreased after treatment with the 3 kinds of stem cell supernatants, and the expression levels under BMSC supernatant 
A

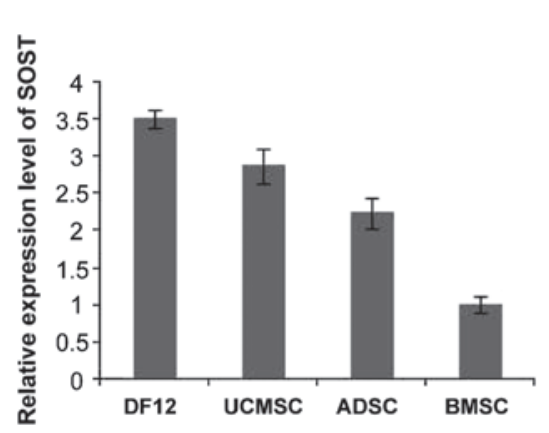

C

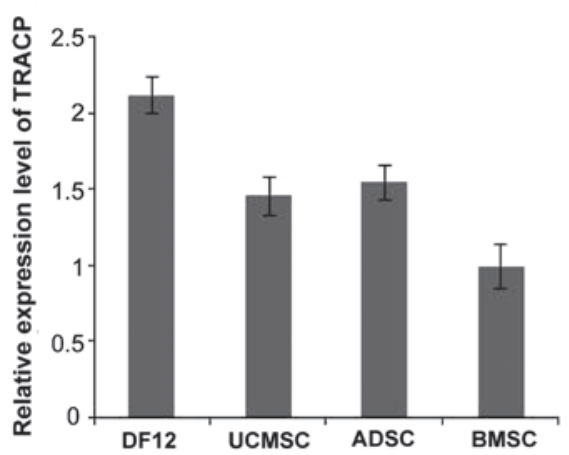

B

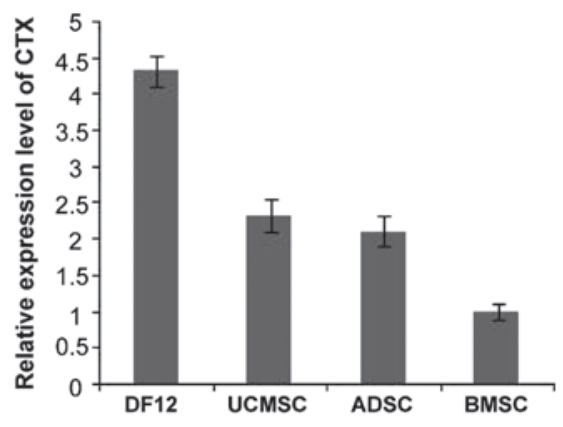

D

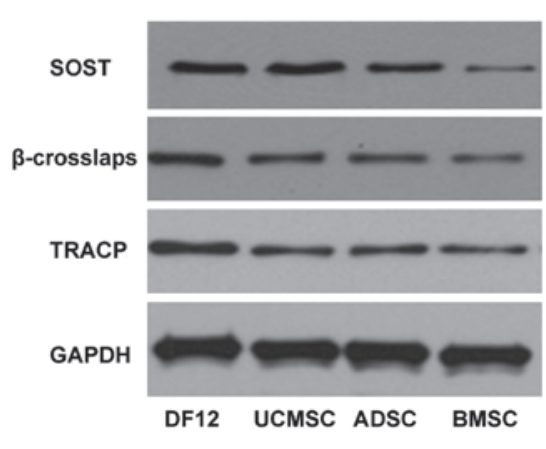

Figure 5. Effect of MSC supernatant on the expression of osteogenesis-inhibiting factors in bone defects. (A-C) Detection of the relative expression levels of SOST, CTX and TRACP via qPCR. (D) Detection results of western blotting. SOST, sclerostin; CTX, collagen carboxy-terminal telopeptide; TRACP, tartrated resistant acid phosphatse.

treatment were the lowest. MSC supernatant, especially BMSC supernatant, could inhibit the expressions of cytokines related to the bone loss, thereby reducing the bone loss at the bone defect and promoting the bone defect healing (Fig. 5).

\section{Discussion}

The treatment of bone defect has always been a problem faced by clinical orthopedists. There are many reasons for bone defects, including both external trauma and internal infection (11). Although the body itself has the wound repair mechanism, the larger wound defect can not be healed by itself (12). At present, bone grafting is commonly used to treat the large bone defect (13), but it still has some problems, the application of autologous bone grafting is limited by the limited bone supply in the donor site, increased surgical risk and prolonged surgery time (14). Allogeneic bone grafting may cause the rejection reaction of body, disease transmission, nonunion and other complications, which also limits the clinical application of allogeneic bone grafting (15).

MSCs belong to the pluripotent cells, and they are derived from the mesoderm and ectoderm in the early development, hence comes the name (16). It was first discovered in the bone marrow, namely BMSC, which not only has the differentiation function of embryonic stem cells, but also can be replicated, cultured and cryopreserved on a large scale in vitro (17). It can differentiate into a variety of required functional cells through the induction of chemical substances and bioactive factor, thus forming the functional tissues and organs. The current known MSCs can be transformed into up to 10 kinds of cells, so it has a very high application value and prospect in the tissue and organ repair (18). At present, the commonly-used MSCs mainly include BMSCs, UMSCs and AMSCs.

Stem cell supernatant was first studied in the embryonic stem cells. The protein multi-dimensional electrophoresis and cytokine antibody chip analysis reveal that there are 201 kinds of independent gene products in the supernatant cultured in human stem cells, more than $80 \%$ of which can be detected via the quantitative reverse transcription-polymerase chain reaction (RT-PCR). The function of stem cell supernatant can be exerted in the metabolism, defense response and tissue differentiation, which can activate the signaling pathways, including vascular biology, bone development and other transforming growth factors. There are many cytokines that can promote the cell growth in the stem cell supernatant, including epidermal growth factor (EGF) and fibroblast growth factor (FGF). The proteomics analysis of stem cell medium provides more experimental basis for the paracrine mechanism of stem cells (19). At present, there are many studies on the effect of MSC supernatant on the healing of skin injury, but its effect on bone defects has not been reported.

BMP-2, as an important marker of bone formation, exists and plays a role in multiple signaling pathways related to the bone formation and regeneration, which can enhance the production of proteins required for bone tissues and strongly promote the differentiation of cells with differentiation potential into the osteoblasts. Some studies considered that BMP-2 is one of the most valuable index and growth factors in the bone formation (18), which is necessary in the differentiation of MSCs into osteocytes. OCN is a kind of vitamin K-dependent cadherin, which plays an important role in the bone mineralization. On one hand, it strengthens the reaction of calcium 
ions with hydroxyapatite in the body; on the other hand, it suppresses the abnormal deposition after the combination of them, thus benefiting the normal development and formation of bone. Once the synthesis of OCN in the body is blocked, it will affect the normal combination of calcium ions and hydroxyapatite, thus hindering the formation of osteoclasts. Therefore, OCN is often used as an important marker of osteoblasts. ALP is also a unique substance expressed by osteoblasts during bone formation, whose concentration can often reflect the capacity of bone formation, because it can bind to the hydroxy limestone via the hydrolyzing phosphate and accelerate its precipitation, thereby increasing the rate of bone formation.

SOST (sclerosteosis) gene can express the SO (sclerostin), the latter of which promotes the osteoblast apoptosis. Studies have found that SO can promote the expressions of a variety of apoptosis factors, and can modify BMP with phosphorylation, thus inhibiting the activity of protein factor BMP that promotes the osteoblast conversion, and blocking the bone formation. Type I CTX can improve the activity of osteoclasts and strengthen the bone resorption and transformation, leading to the gradual degradation of loss of bone, and causing osteoporosis, osteomalacia, fractures and other metabolic diseases. TRACP is also an important marker of bone resorption, whose level is also high in the blood when the osteoclast activity is high. TRACP can increase the OCN activity, often accompanied by osteoporosis, osteomalacia and bone metastatic cancer (20).

In conclusion, the data of three kinds of typical MSCs show that the culture supernatant of MSCs contains the active substances that can promote the bone defect repair, which can also increase the high expression of osteocyte formation factors and inhibit the low expression of the factors related to the bone loss through the signaling pathway, thereby promoting the bone defect repair.

\section{References}

1. Li C, Zhang W, Jiang X and Mao N: Human-placenta-derived mesenchymal stem cells inhibit proliferation and function of allogeneic immune cells. Cell Tissue Res 330: 437-446, 2007.

2. Bailo M, Soncini M, Vertua E, Signoroni PB, Sanzone S, Lombardi G, Arienti D, Calamani F, Zatti D, Paul P, et al: Engraftment potential of human amnion and chorion cells derived from term placenta. Transplantation 78: 1439-1448, 2004.

3. Yasko AW, Lane JM, Fellinger EJ, Rosen V, Wozney JM and Wang EA: The healing of segmental bone defects, induced by recombinant human bone morphogenetic protein (rhBMP-2). A radiographic, histological, and biomechanical study in rats. J Bone Joint Surg Am 74: 659-670, 1992.

4. Rose FR and Oreffo RO: Bone tissue engineering: Hope vs. hype. Biochem Biophys Res Commun 292: 1-7, 2002.
5. Fleming JE Jr, Cornell CN and Muschler GF: Bone cells and matrices in orthopedic tissue engineering. Orthop Clin North Am 31: 357-374, 2000.

6. Kimelman N, Pelled G, Gazit Z and Gazit D: Applications of gene therapy and adult stem cells in bone bioengineering. Regen Med 1: 549-561, 2006.

7. Mobasheri A, Csaki C, Clutterbuck AL, Rahmanzadeh M and Shakibaei M: Mesenchymal stem cells in connective tissue engineering and regenerative medicine: Applications in cartilage repair and osteoarthritis therapy. Histol Histopathol 24: 347-366, 2009.

8. Lazáry A, Balla B, Kósa JP, Bácsi K, Nagy Z, Takács I, Varga PP, Speer G and Lakatos P: Effect of gypsum on proliferation and differentiation of MC3T3-E1 mouse osteoblastic cells Biomaterials 28: 393-399, 2007.

9. Zhu SF, Zhong ZN, Fu XF, Peng DX, Lu GH, Li WH, Xu HY, $\mathrm{Hu} \mathrm{HB}$, He JM, Su WY, et al: Comparison of cell proliferation, apoptosis, cellular morphology and ultrastructure between human umbilical cord and placenta-derived mesenchymal stem cells. Neurosci Lett 541: 77-82, 2013.

10. Qiao C, Xu W, Zhu W, Hu J, Qian H, Yin Q, Jiang R, Yan Y, Mao F and Yang H: Human mesenchymal stem cells isolated from the umbilical cord. Cell Biol Int 32: 8-15, 2008.

11. Park BS, Choi BG and Park $\mathrm{CH}$ : Mass produing method of growth factor using adipose derived adult stem cells. US Patent EP1974022 A4. Filed October 12, 2006; issued December 16, 2009.

12. Caverzasio J: Strontium ranelate promotes osteoblastic cell replication through at least two different mechanisms. Bone 42: 1131-1136, 2008

13. Diefenderfer DL, Osyczka AM, Garino JP and Leboy PS: Regulation of BMP-induced transcription in cultured human bone marrow stromal cells. J Bone Joint Surg Am 85-A (Suppl 3): 19-28, 2003.

14. Coelho MJ and Fernandes MH: Human bone cell cultures in biocompatibility testing. Part II: Effect of ascorbic acid, beta-glycerophosphate and dexamethasone on osteoblastic differentiation. Biomaterials 21: 1095-1102, 2000.

15. Kadiyala S, Jaiswal N and Bruder SP: Culture-expanded, bone marrow-derived mesenehylllal stem cells can regenerate a critieal-sized segmental bone defect. Tissue Eng 3: 173-185, 2007.

16. Gangji V and Hauzeur JP: Cellular-based therapy for osteonecrosis. Orthop Clin North Am 40: 213-221, 2009.

17. Cheng SL, Lou J, Wright NM, Lai CF, Avioli LV and Riew KD: In vitro and in vivo induction of bone formation using a recombinant adenoviral vector carrying the human BMP-2 gene. Calcif Tissue Int 68: 87-94, 2001.

18. Riley EH, Lane JM, Urist MR, Lyons KM and Lieberman JR: Bone morphogenetic protein-2: Biology and applications. Clin Orthop Relat Res 324: 39-46, 1996.

19. Fukuchi Y, Nakajima H, Sugiyama D, Hirose I, Kitamura T and Tsuji K: Human placenta-derived cells have mesenchymal stem/progenitor cell potential. Stem Cells 22: 649-658, 2004.

20. Baksh D, Yao R and Tuan RS: Comparison of proliferative and multilineage differentiation potential of human mesenchymal stem cells derived from umbilical cord and bone marrow. Stem Cells 25: 1384-1392, 2007.

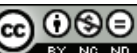

This work is licensed under a Creative Commons Attribution-NonCommercial-NoDerivatives 4.0 International (CC BY-NC-ND 4.0) License. 